\title{
Low surface brightness galaxies mass profiles as a consequence of galactic evolution.
}

\author{
C. Alard \\ Institut d'Astrophysique de Paris, 98bis boulevard Arago, 75014 Paris \\ e-mail: alard@iap.fr
}

\begin{abstract}
Aims. This paper presents a principal components analysis of rotation curves from a sample of low surface brightness galaxies. The physical meaning of the principal components is investigated, and related to the intrinsic properties of the galaxies.

Methods. The rotation curves are re-scaled using the optical disk scale, the resulting principal component decomposition demonstrates that the whole sample is properly approximated using two components.

Results. The ratio of the second to the first component is related to the halo steepness in the central region, is correlated to the gas fraction in the galaxy, and is un-correlated to other parameters. As a consequence the gas fraction appear as a fundamental variable with respect to the galaxies rotation curves, and its correlation with the halo steepness is especially important.

Conclusions. Since the gas fraction is related to the degree of galaxy evolution, it is very likely that the steepness of the halo at the center is a consequence of galaxy evolution. More evolved galaxies have shallower central profile and statistically less gas, most likely as a consequence of more star formation and supernovae. The differences in evolution, gas fractions and halo central steepness of the galaxies could be due to the influence of different environments.
\end{abstract}

Key words. Cosmology:dark matter,Galaxies:evolution,Galaxies:dwarf

\section{Introduction.}

Cold dark matter predicts steep density profile at the center of dark matter halo's (Navarro, Frenk \& White 1996; Navarro, Frenk \& White 1997; Moore, et al., 1998). This prediction is not consistent with observations of constant core density in low surface brightness galaxies (Moore, 1994; Burkert, 1995; Navarro, Frenk \& White 1996; McGaugh \& de Blok, 1998; de Blok \& Bosma 2002). Various modifications of the cold dark matter scenario have been proposed to account for this discrepancy, collisional dark matter (Carlson, Machacek, \& Hall, 1992; Spergel \& Steinhardt, 2000; Moore et al., 2000), Fluid dark matter (Peebles 2000), Boses Einstein condensates (Hu, Barkana, \& Gruzinov). Other approaches do not attempt to modify the cold dark matter scenario, but emphasize the role of supernovae, ram pressure stripping or tidal interactions as mechanism affecting the mass profile of 
dwarf galaxies (Read \& Gilmore 2005). This paper will not attempt to favor one or another scenario, but will focus on analyzing rotation curves data without preconceived hypothesis. The approach will focus on isolating the fundamental features in the rotation curves and will relate them to the intrinsic physical parameters of low surface brightness galaxies (LSB).

\section{Data analysis.}

de Blok \& Bosma (2002), (hereafter dBB) published the rotation curves of 26 LSB. The dBB sample contains galaxies of different sizes and masses requiring the re-scaling of the rotations curves. In practice, the rotation curves were re-scaled using the length scale inferred from the visible light (Table 1 in $\mathrm{dBB}$ ). Provided the various rotation curves are related to the same universal physical process, the re-scaled curves should be reducible to a minimal set of fundamental components. The reduction to a minimal set is equivalent to a principal component analysis (PCA). The PCA requires a re-sampling of the curves to a common grid, this re-sampling was performed using B-spline interpolation. The PCA vectors are re-constructed in the range $R_{p c}$ (in re-scaled units). The range $R_{p c}$ must include a sufficient number of points (a minimum of 5 points was required). An additional requirement was the availability of an estimation of the $\mathrm{HI}$ contribution to the rotation curve. The contribution of the gas was subtracted to the mass budget. The range $R_{p c}$ was adjusted to be as large as possible while maximizing the number of curves. The optimal value is $R_{p c}=2.85$, which corresponds to a total of 15 curves. Since the mass scale like the square of the velocity the PCA is performed on the square of the velocity data. Finally the amplitudes of the curves were re-normalized so that their self cross product is unity. The PCA matrix is constructed by filling the matrix columns with the rotation curve vectors. $\mathrm{PCA}$ requires to find the eigenvalues of the covariance matrix, numerically this is best achieved by using a singular value decomposition of the matrix. Components are sorted by descending order of power spectrum contribution (see Fig. 1).

\subsection{Effect of noise on the PCA analysis.}

Numerical experiments performed using Monte-Carlo simulations with gaussian noise expectations on the velocities (as provided by $\mathrm{dBB}$ ) indicates that the amplitude of the noise on the power spectrum of each components (sums of the square of amplitudes for each vector, normalized by total power), is about $(0.035)^{2}$. As a consequence only the first two components are significant (see Fig. 1, 2). Another problem is the stability of these two components with respect to the noise fluctuations and the re-scaling errors. Random gaussian noise with variance taken from $\mathrm{dBB}$ were added to the set of light curves and the singular value decomposition was re-computed each time, in order to estimate the effect of noise on the principal components. The result of 1000 simulations shows that the noise accounts for about $0.5 \%$ of the total variance on the first component, and $15 \%$ of the total variance on the second component. These numerical experiments shows also that only a linear interpolation of the rotation curves is stable numerically. Let's now investigate the errors introduced by the re-scaling of the rotation curves. The curves were re-scaled 
according to the disk scale length, but there is no guaranty that the scale of the other components are exactly proportional to the disk scale. To evaluate the effects of uncertainty in the re-scaling, uniform random fluctuations of the scale length were introduced and the PCA was re-conducted each time. The results shows that for uniform deviations of the scale length with respective amplitudes of, $10 \%, 20$, and $40 \%$, the corresponding variance of the noise as a fraction of the total variance of the second components is: $2 \%, 4 \%$, and $9 \%$. In addition to these results note that the mean correlation coefficients between the simulated second component and the original one is 0.9 when noise fluctuations are considered, and higher for scale fluctuations. The first components is much less affected and has a mean correlation close to unity. As a conclusion the results on the statistical variance, and the correlation are a good illustration of the stability of the PCA analysis of this sample.

\subsection{Interpretation of the components.}

The first component represents approximately the mean of all curves. The meaning of the second component is easily understood by evaluating its effect on the shape of the curves. Adding a fraction of the second component to the first component influence the power law approximation of the curve. Adding $20 \%$ of the second component to the first component corresponds to a power law approximation with exponent $\simeq 1.5$, while subtraction the same amount corresponds to an exponent of $\simeq 0.5$. As a consequence the ratio of the second component to the first component is also related to the steepness of the density profile, or equivalently to the concentration of mass at the center. This analysis is confirmed by the strong correlation observed between the mass concentration (ratio of mass to 1 scale unit to mass at 3 scale units) and the ratio between the components. As a consequence the variations in shape of the rotation in the $\mathrm{dBB}$ sample are related to a variation in the concentration of mass at the center.

\section{Relation between rotation curve and and galaxy physics.}

The rotation curves of the $\mathrm{dBB}$ sample are properly approximated with two PCA components, and the ratio of these components $\eta$ is associated with the concentration of mass at the center. Let's now investigate the relation between $\eta$ and the other galaxies parameters, magnitude $M_{R}$, surface brightness $\mu_{R}$, scale $h_{R}$, mass of gas $M_{G}$, and total mass $M$. A significant correlation is found between the gas fraction $\log (G / M)$ and $\eta$. No significant correlation is found between $\eta$ and other variables (see table 1). The statistical significance of the correlation between the variables are analyzed using 2 different estimates, the Spearman correlation coefficient, and the a linear least-square fit (with errors in both directions). To evaluate the significance of the correlation, the probability that the Spearman correlation coefficient is different from zero is computed. However the problem is complicated by the fact that each point of a given curve has a different variance. Thus the Spearman correlation analysis should be considered as an evaluation of the real correlation between the variable. Another type of analysis that would take into account the different variance of the data points is to fit a straight line, and estimate the statistical significance that the slope is different from zero. The statistical errors on the different variable and $\eta$ 


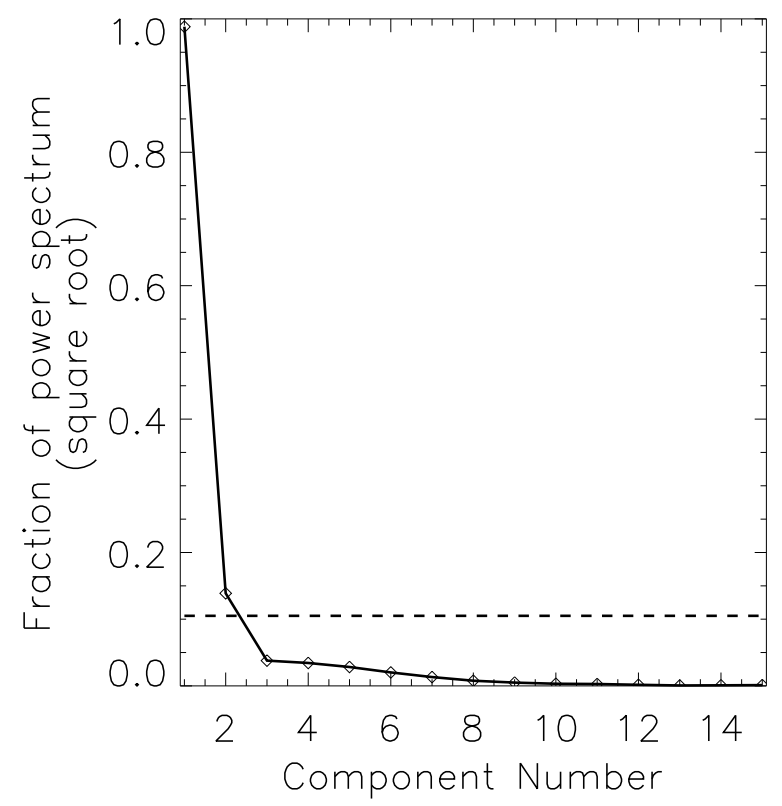

Fig. 1. Square root of the power spectrum contribution for each principal component in order of decreasing contribution. The dotted line represents the $3-\sigma$ level of the noise expectation.

are computed using the initial errors on the light curves data points from dBB. A Least square minimization with errors in both variable is conducted (Press 2007). The chi-square of the fit is about $\simeq 3$, which is large and indicates that other errors, probably due to to an intrinsic noise in the relation between the variables are present. It is assumed that this intrinsic noise amplitude $\sigma$ is constant whatever the galaxy. The value of $\sigma$ is unknown and must be estimated from the data. A rough estimate of $\sigma$ can be obtained by requiring that the chi-square is reduced to unity for the relevant value of $\sigma=\sigma_{0}$. The real value of $\sigma$ must be close to $\sigma_{0}$ but in practice $\sigma$ is unknown and the full space of possible realizations has to be explored, by running a large number of numerical simulations for all values of $\sigma$. Among the full space of possible realizations, one has to select the sub-set of simulations which is consistent with the data. A simple measure of the consistency is that the reduced chi-square, $\chi^{2}$ is equal to unity when fitting a straight line and considering an intrinsic noise with amplitude $\sigma_{0}$. In practice the sub-set of simulations consistent with the data is selected using the criteria, $\left|\chi^{2}-1\right|<0.01$. The statistical distribution of the slope for the sub-set is well approximated by a gaussian, with a standard deviation $\alpha$. The statistical significance of the slope $b$ is evaluated by computing the ratio, $b / \alpha$ (see Table 1). The association between $\eta$ and the gas fraction is very significant, both in the Spearman correlation coefficient (1.6\% chance of no association between $\eta$ and the gas fraction), and the straight line fit $(3.5 \sigma)$. There is no significant association of other variables with $\eta$. Is the association between $\eta$ and the gas fraction real or is it due to any systematic effect ? One possibility would be that the association is indirect and reflects an association of each variable with another variable. However since there is no correlation between the second variable and any other variables except $\eta$ this hypothesis can be rejected. Another point 

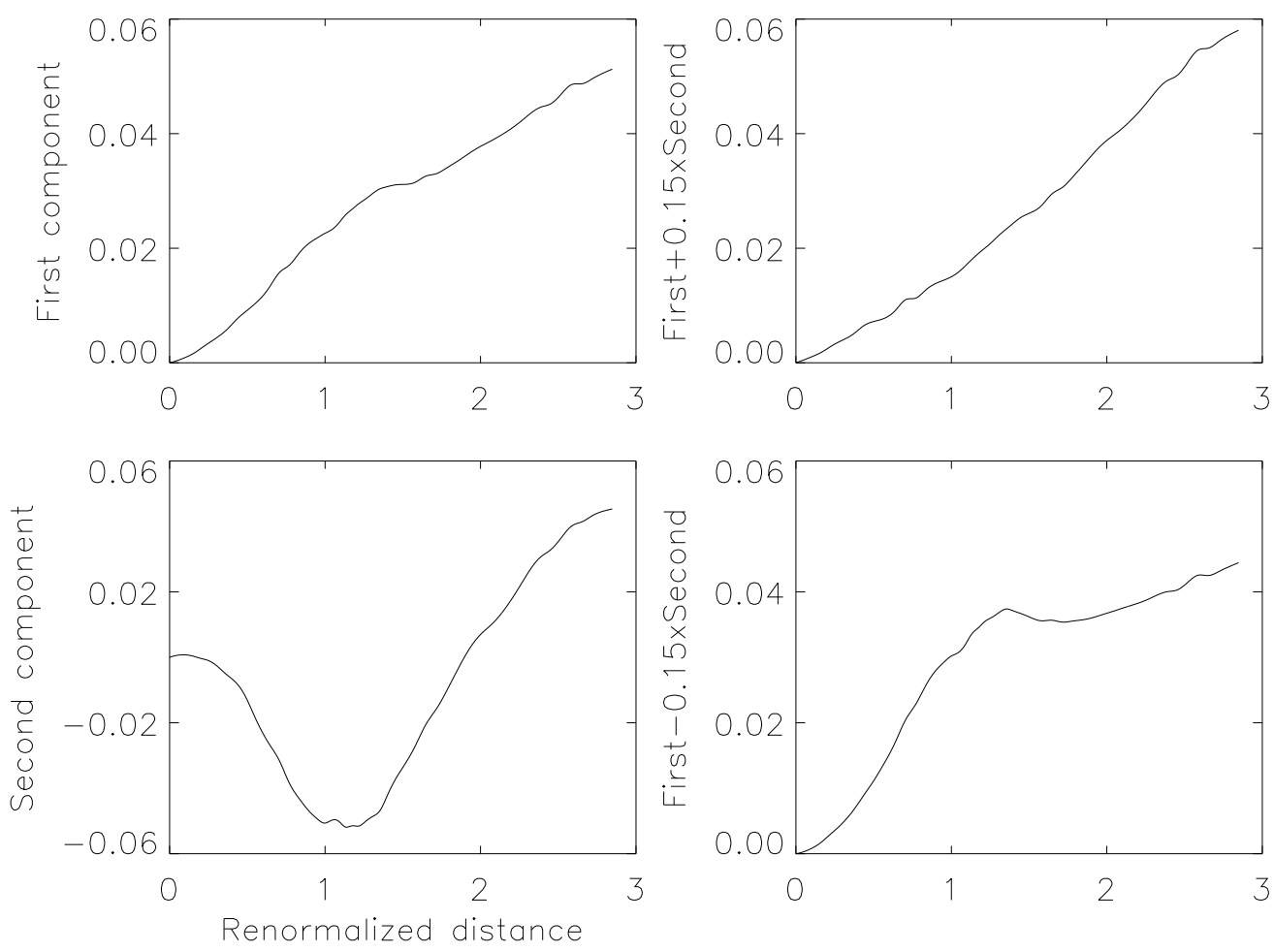

Fig. 2. The first two components presented together with linear combinations of the components illustrating the effect of the second component on the rotation curves.

is that the mass budget considered in this analysis include also the stellar disk. Does the contribution from the stellar disk introduces a correlation in the variable that would be mistakenly attributed to the dark halo ? The correlation that we observe is that galaxies with larger gas fractions are more centrally concentrated. On the other hand the gas is converted into stars, thus a higher gas fraction is expected to corresponds to lower stellar content. As a consequence in galaxies with a higher gas fraction the stellar disk is expected to contribute less to the mass budget. Thus the effect of the stellar disk is in an opposite direction with respect to the observed correlation between the gas fraction and $\eta$. It is interesting to point that the assumed correlation between higher gas fraction and lower stellar fraction $(S / M)$ is confirmed by analyzing the dBB sample. The Pearson correlation coefficient between the stellar fraction and the gas fraction is is -0.53 , which corresponds to a chance of only $4 \%$ that the variable are un-correlated. Note that to derive the stellar fraction, the stellar mass was normalized by a mean statistical mass $M_{S}$ for the galaxy which was derived by fitting the data: $M_{S} \propto h_{R}^{1.6}$. This normalization was preferred to a normalization by the total mass extrapolated from the rotation curve because it introduces less noise. Fitting a straight line using the procedure already described for the gas fraction and $\eta$ demonstrates that the association is significant to more than $3 \sigma$. Note also that by taking into account the relation between the gas fraction and the stellar fraction and the relation between the gas fraction and $\eta$ one can infer the relation between the stellar fraction and $\eta$. The inferred slope is consistent with the data, no correlation is found between the data, but this is due to the fact that inferred slope is small with respect to the noise. 
Table 1. Correlation and significance of the correlation between different variables and $\eta$. The lower line presents the results obtained by fitting a straight line to the same set of variables.

\begin{tabular}{cccccc} 
& $\log \left(M_{G} / M\right)$ & $M_{R}$ & $\mu_{R}$ & $\log \left(h_{R}\right)$ & $\log (S / M)$ \\
\hline Correlation & -0.61 & 0.03 & -0.28 & -0.18 & 0.057 \\
Significance & 0.016 & 0.91 & 0.32 & 0.51 & 0.84 \\
\hline Fit Slope & -0.31 & 0.03 & -0.41 & -0.07 & -0.017 \\
$\mid$ Slope $\mid / \sigma$ & 3.5 & 0.64 & 0.74 & 0.94 & 0.15 \\
\hline
\end{tabular}

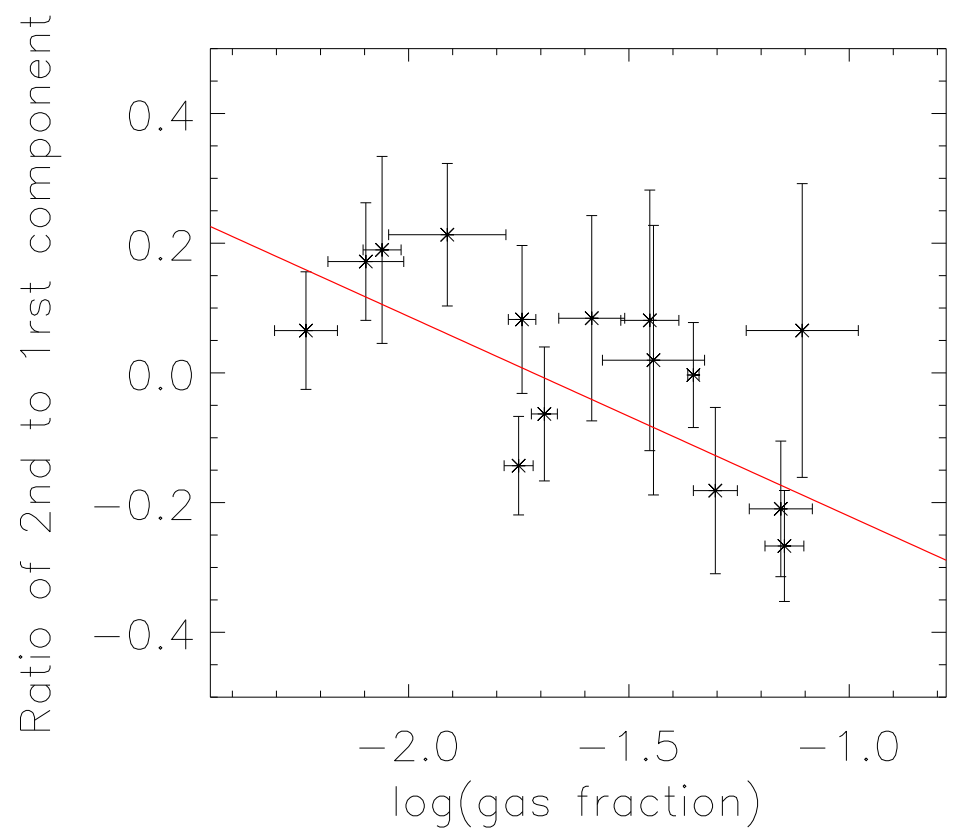

Fig. 3. The ratio of the second to the first principal component as a function of the gas fraction. Note that negative values of $\eta$ corresponds to more centrally concentrated mass profiles.

\section{Discussion}

The shape of galaxy rotation curve and the mass concentration at the center are related to $\eta$. Furthermore, $\eta$ is related to the the gas fraction in the dBB sample. The parameter $\eta$ is also closely related to mass model fitting of the rotation curves (see Fig. 4). Negative values of $\eta$ are associated with more concentrated mass profile, shorter Burkert scale length and better consistency with a NFW profile when compared to positive values of $\eta$. As a consequence gas rich galaxies will be also associated with shorter Burkert scale and will be closer to a NFW profile. Note that this association cannot be due to a systematic bias related to the mass of the galaxies. One could think that more massive galaxies have steeper profile and more gas because the self gravity is stronger and more easily overcome the supernovae winds. However since no correlation between mass and $\eta$ is observed this systematic bias is unlikely. Actually the effect of stronger gravity in more massive system could be compensated by more efficient star formation. Kim (2007) found that the gas 
mass fraction is correlated to the ratio of current to past average star formation. The results of Kim (2007) shows that the gas fraction is related to the evolution of galaxies, gas poor galaxies had more stellar formation in the past than gas rich galaxies with respect to current star formation. Statistically the gas rich galaxies are less evolved, have lower stellar fractions, and have a better consistency with NFW profiles. Provided that the initial dark matter profile is close to a NFW profile the effect of galaxy evolution via supernovae explosions is to form shallower profiles with nearly constant density cores (Larson 1974, Read \& Gilmore 2005, Governato et al. 2010). The effect of tidal encounter may boost galaxy evolution and influence the mass profile (Mayer et al 2001, Hayashi et al 2003). Galaxies interaction may also increase the star formation rate and influence the stellar mass function (Habergham et al 2010). Star formation, Galaxy interactions and other effects related to galaxy evolution flattens the inner mass profile, and in the same time reduce the gas fraction fraction in the galaxy. The relation between mass profile and gas fraction is thus most likely a correlation between galaxy evolution and mass profile. We observe an evolutionary sequence where galaxies with a larger gas fraction are less evolved than gas poor galaxies. It is likely that gas poor galaxies were subject to more interactions with other galaxies, boosting stellar formation and supernovae, while at the same time the tidal interaction has also some influence on the mass profile. An analysis of SDSS data by Rosenbaum \& Bomans (2004) shows that gas rich LSB forms in the voids and that some migrated to the edge of filaments while others remain in the voids. This scenario implies that LSB galaxies had less tidal interaction than other brighter galaxies, but the level of tidal interaction encountered by a given LSB galaxy is as variable as the neighborhood of the galaxy is, even if they form in relatively void regions, some encounters are still likely, and those galaxies who later fall on more densely populated areas receive more tidal interaction than others. This would explain the differences of evolution in this sample, and the corresponding differences in the rotation curves and mass profiles. +

\section{References}

de Blok, W., Bosma, A., 2002, A\&A, 385, 816

Burkert, A., 1995, ApJ, 447, L25

Carlson, E., Machacek, M., Hall, L., 1992, ApJ, 398, 43

Governato, F., Brook, C., Mayer, L., Brooks, A., Rhee, G., Wadsley, J., Jonsson, P., Willman, B., Stinson, G., Quinn, T., Madau, P., 2010, Nature, 463, 203

Habergham, S., Anderson, J., James, P., 2010, ApJ, 717, 342

Hayashi E., Navarro J. F., Taylor J. E., Stadel J., Quinn T., 2003, ApJ, 584, 541

Hu, W., Barkana, R., Gruzinov, A., 2000, PhRvL, 85, 1158

Kim, Ji Hoon, Ph.D. dissertation, The star formation history of low surface brightness galaxies, 2007

Larson, R., 1974, MNRAS, 169, 229

Mayer L., Governato F., Colpi M., Moore B., Quinn T., Wadsley J., StadelJ., Lake G., 2001, ApJ, 547, L123

McGaugh, S., de Blok, W., 1998, ApJ, 499, 41

Moore, B., Gelato, S., Jenkins, A., Pearce, F., Quilis, V., 2000, ApJ, 535, L21

Moore, B., Governato, F., Quinn, T., Stadel, J., Lake, G., 1998,,ApJ, 499, L5

Moore, B., 1994, Nature, 370, 629

Navarro, J. Frenk, C., White, S., 1997, ApJ, 490, 493

Navarro, J. Frenk, C., White, S., 1996, ApJ, 462, 563 


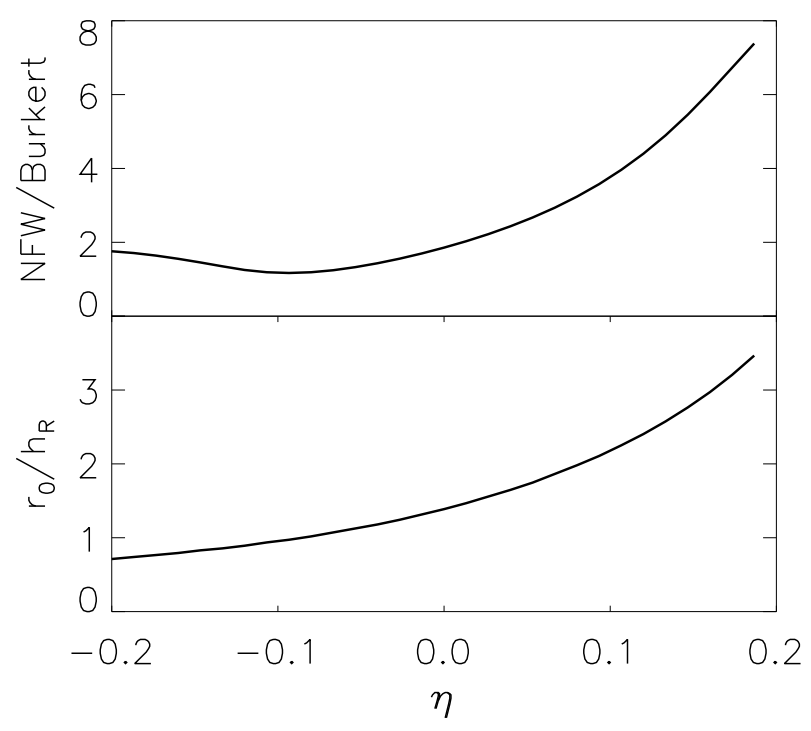

Fig. 4. Fit of Burkert and NFW profiles to a linear combination of the first and second components. Negative values of $\eta$ corresponds to more centrally concentrated profiles. The lower plot presents the scale length of the fitted Burkert profile $r_{0}$ as a function of $\eta$. The upper plot presents the ratio of the residual obtained by fitting a NFW profile to the residual obtained by fitting a Burkert profile.

Peebles, J., 2000, ApJ, 534, L127

Press, W., Teukolsky, S., Vetterling, W., Flannery, B., Numerical Recipes, Cambridge University Press, 2007

Spergel, D., Steinhardt, P., 2000, PhRvL, 84, 3760

Read, J., Gilmore, G., 2005, MNRAS, 356, 107

Rosenbaum, S., Bomans, D., 2004, A\&A, 422L, 5

Wechsler, R., Bullock, J., Primack, J., Kravtsov, A. Dekel, A.,2002, ApJ, 568, 52 